\title{
Abstract \\ Clinico-epidemiological study of Melasma in a Private medical center at Anuradhapura district for 6 month period.
}

Weerakoon $\mathrm{H}^{1 *}$

${ }^{1}$ PDHS Office, Anuradhapura

\begin{abstract}
Background

Melasma is a dark skin discoloration or a patchy brown tan. It is characterized by the development of dark brown spots on the forehead, nasal bridge, cheek, upper lip and neck. The dark spots may be ugly in appearance, but this is a relatively harmless skin condition. Incidence is higher in women and onset occur around 3rd or 4th decades. The causative factions for melasma are hormonal imbalance, sun exposure, genetics, thyroid disease and allergic conditions. It is a condition of major social and cosmetic concern in Sri Lanka. Objectives of this study were to analyze the epidemiology and clinical features and to determine associated factors of Melasma.

Method

Observational study was conducted in 92 patients in a private medical center in Anuradhapura for 6 months duration from October 2014 to April 2015. Demographic data, family history, beliefs about the disease and associated factors were recorded.

\section{Results}

Melasma is commoner in women with male to female ratio of 1:7.3. Age of onset was 41 to 50 years in $48.9 \%$. Involvement of cheek is the commonest presentation $(63 \%)$ followed by cheek, forehead and nose involvement $(17.3 \%)$, cheek and nose involvement (11.9\%) and cheek and forehead involvement (7.6\%). Melasma is more common in fair skinned individuals (73.9\%). 27\% had family history of similar lesions. Melasma is associated with usage of hormonal contraceptive in $13.5 \%$ of women. $78 \%$ of woman had associated under eye pigmentation and $31.5 \%$ had history of exposure to sunlight. None had thyroid disease or allergic history.

\section{Conclusions}

Melasma is common in women. Under eye pigmentation is the most common association.
\end{abstract}

Key words: Melasma; Clinico Epidemiological Study; Private Medical Center

Copyright:(C) 2015 Weerakoon $\mathrm{H}$ et al. This is an open access article distributed under the Creative Commons Attribution License, which permits unrestricted use, distribution, and reproduction in any medium, provided the original work is properly cited.

* Correspondence : hemaweerakoon68@gmail.com

Cite this abstract as: Weerakoon $\mathrm{H}^{1}$. Clinico-epidemiological study of Melasma in a Private medical center at Anuradhapura district during 6 months period. Anuradhapura Medical Journal 2015;9 (2Supp):S43.

DOI: http://dx.doi.org/10.4038/ami.v9i2Supp.7592 


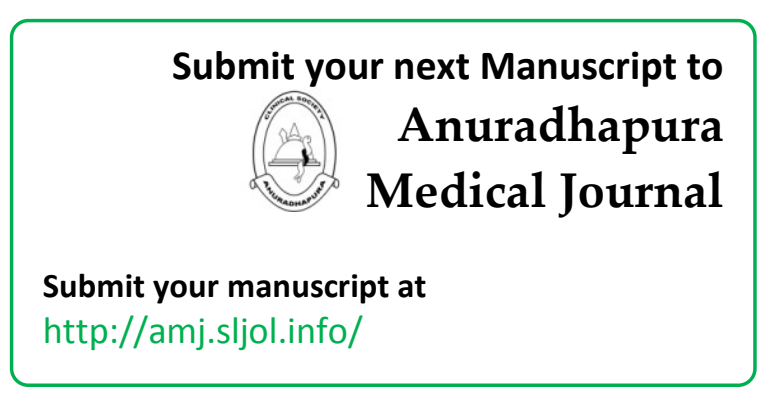

